\title{
Cancer prevention: cervical cancer
}

\author{
Mamsau Ngoma ${ }^{1}$ and Philippe Autier ${ }^{2}$ \\ ${ }^{1}$ Ocean Road Cancer Institute, PO Box 5408, Dar Es Salaam, Tanzania \\ ${ }^{2}$ International Prevention Research Institute, Espace Européen d'Ecully, Bâtiment G, Allée Claude Debussy, 69130 Ecully ouest Lyon, France
}

\section{Abstract}

Cervical cancer is the fourth most common cancer in women, and the seventh overall, with an estimated 528,000 new cases and 266,000 deaths in 2012 [1]. Almost nine out of ten (87\%) cervical cancer deaths occur in the less-developed regions of the world. The cervical cancer incidence significantly increases after 20 years of age and peaks at 50 years of age. Because cervical cancer mainly affects African women at a relatively young age, the socio-economic consequences are enormous. The human papillomavirus (HPV) is central to the development of cervical neoplasia and can be detected in $99.7 \%$ of cervical cancers. Hence primary prevention aims at reducing human papillomavirus (HPV) infection by HPV vaccine administration. Secondary prevention involves cervical cancer screening and management of precancerous lesions via either Pap smear, visual inspection with acetic acid (VIA) or with lugols iodine (VILI) or HPV testing for high-risk HPV types.

Conclusion: Sub-Saharan countries still have a long way to go in controlling the high burden of cervical cancer. Effective prevention methods exist, such as HPV vaccination and screening, but their affordability and implementation remain challenging for most of these countries. Despite that, there is still light on the horizon, as the cost of HPV vaccines has been steadily decreasing and most African countries are using the more costeffective methods of cervical cancer screening.

Keywords: cervical cancer, prevention, screening, HPV, vaccination

\section{Background}

Cervical cancer is the fourth most common cancer in women, and the seventh overall, with an estimated 528,000 new cases and 266,000 deaths in 2012 [1]. Almost nine out of ten (87\%) cervical cancer deaths occur in the less-developed regions. Although the registration of cancer cases and of cancer deaths is uncommon in Africa, it is estimated that about one-fifth of the world burden of cervical cancer cases and of deaths would occur in Africa [2]. The disease is unevenly distributed, with peak incidences of 30 cases and more per 100,000 women per year in the central and eastern part of the continent, and in countries surrounding the Guinea Gulf [3]. In contrast, the incidence would be less than 10 cases per 100,000 women per year in African countries located around the Mediterranean Sea.
Correspondence to: Mamsau Ngoma Email: mamsaungoma@yahoo.com

ecancer 2019, 13:952

https://doi.org/10.3332/ecancer.2019.952

Published: $25 / 07 / 2019$

Received: 08/11/2018

Publication costs for this article were supported by ecancer (UK Charity number 1176307).

Copyright: (c) the authors; licensee ecancermedicalscience. This is an Open Access article distributed under the terms of the Creative Commons Attribution License (http:// creativecommons.org/licenses/by/3.0), which permits unrestricted use, distribution, and reproduction in any medium, provided the original work is properly cited. 


\section{Socio-economic burden of cervical cancer}

The cervical cancer incidence significantly increases after 20 years of age and peaks at 50 years of age. Because cervical cancer mainly affects African women at a relatively young age, the socio-economic consequences are enormous. So, women in sub-Saharan Africa lose more years to cervical cancer than to any other type of cancer. Many women with cervical cancer can no longer take care of their family, and their death leaves many orphans.

\section{The cause of cervical cancer}

There are two major histologic types of cervical cancer, squamous cell carcinoma (about 75\%) which mostly starts at the transformation zone of the ectocervix and adenocarcinoma (about 25\%) which arises in the glandular columnar layer of the endocervix. The human papillomavirus (HPV) is central to the development of cervical neoplasia and can be detected in 99.7\% of cervical cancers [4]. It is mostly caused by chronic infection with high-risk strains of HPV (mainly the subtypes 16 and 18). The HPV subtypes associated with squamous cancer are different from those associated with adenocarcinoma [5].

\section{Risk factors}

HPV infection is essentially a sexually transmitted infection (STI), and therefore many risk factors for cervical cancer are those associated with a higher risk of STI occurrence. Factors that increase HPV acquisition or transmission, and promote the oncogenic effect of the virus are widespread in Africa. These are:

- young age at marriage or first sexual intercourse (i.e., before age 15 or 16 years),

- a high number of sexual partners,

- polygamy,

- a partner that has sexual intercourse with other partners,

- unprotected sexual intercourse (e.g., non or infrequent use of condoms, diaphragms or gels),

- a history of sexually transmitted infections or diseases,

- low education, deprivation,

- non-attendance or non-access to cervical cancer screening.

In addition to these factors, an impaired ability to clear HPV infection is probably the strongest risk factor for cervical cancer in Africa. Impairment of the immune system due to being infected with human immunodeficiency virus (HIV) is associated with persistent HPV infection which is the primary cause of cervical intra-epithelial neoplasia that may progress into an invasive cancer if left untreated. African HIV-positive women would have a 2 to 12 -fold higher risk of intraepithelial neoplasia (CIN) lesions compared with HIV-negative women [6, 7]. Hence, HIV-infected women without overt AIDS, and women with AIDS, are at increased risk for the development of cervical cancer. Cervical cancer occurs at a younger age among HIV-infected women, and the disease tends to be more aggressive than in non-HIV-infected women [8].

\section{Primary prevention in cervical cancer}

\section{Human papillomavirus vaccination}

There are three different vaccines, which vary in the number of HPV types they contain and target, although not all are available in all locations:

- Quadrivalent HPV vaccine (Gardasilß) targets HPV types 6, 11, 16 and 18.

- 9-valent vaccine (Gardasil 9®) targets the same HPV types as the quadrivalent vaccine $(6,11,16$ and 18$)$ as well as types 31,33 , 45, 52 and 58.

- Bivalent vaccine (Cervarix $®)$ targets HPV types 16 and 18. 
The target group for vaccination recommended by the WHO are girls aged 9 to 14 years who have not become sexually active. This is because it has been shown that they have a better immune response to the vaccine than those in their late teens.

The choice of vaccine will depend on the availability and cost. Typically, two doses of HPV vaccine should be given at 0 and at 6 months but for those above 15 years require three doses, the last dose being at 12 months.

The vaccines prevent over 95\% of HPV infections caused by HPV types 16 and 18 and may have some cross-protection against other lesscommon HPV types which cause cervical cancer [9]. HPV vaccination is effective in preventing cervical disease, including cervical intraepithelial neoplasia (CIN2 or 3) and adenocarcinoma in situ. Two of the vaccines also protect against HPV types 6 and 11 which cause anogenital warts. The vaccines work best if administered prior to exposure.

Data demonstrating the high effectiveness of HPV vaccination have been recently released for Scotland. In 2008 , Scotland started a national immunisation programme against HPV using the bivalent vaccine. This programme was school-based and targeted girls aged between 12 and 13 with a catch-up programme until age 18 . Uptake in the routinely immunised cohorts consistently exceeded $85 \%$ with $65 \%$ uptake in the catch-up cohort. Routine vaccination of girls aged 12-13 with the bivalent vaccine in Scotland has led to a dramatic reduction in pre-invasive cervical disease. Compared to un-vaccinated women born in 1988, vaccinated women born in 1995 and 1996 showed an $89 \%$ reduction (95\% Cl: 81\%, 94\%) in prevalent CIN Grade 3 or worse [10].

The safety of anti-HPV vaccination has received great attention. In 2014 , the WHO stated that the two commercially available HPV vaccines 'continued to have an excellent safety profile' [11].

The primary prevention of cervical cancer largely overlaps with the primary prevention of STIs, including that of HIV. In Africa, many studies have been conducted on behaviours and methods associated with lower prevalence and transmission of STIs. Because STIs in general are associated with numbers of sex partners and unprotected sexual intercourse, prevention policies first focused on reductions of sex partners and on the provision of 'barrier methods', such as condoms. Information on the need to reduce high-risk sexual relationships has proven effective in Uganda where this policy has contributed to the control of HIV transmission [12]. Both circumcision and regular condom use are associated with reduced risk for oncogenic and overall HPV [13]. Nevertheless, the incidence of HPV infection among circumcised males and their partners remains high. And the high transmissibility of HPV infection suggests that primary prevention of STIs alone is not sufficient it should go hand in hand with HPV vaccination.

\section{Secondary cervical cancer prevention: cervical cancer screening}

The goal of screening is to decrease the mortality associated with cervical cancer through detecting the disease when still at an early curable stage, or through detecting precursor lesions, i.e., the cervical CIN. The systematic removal of CIN lesion during screening also leads to reductions of the incidence of invasive cervical cancers of all stages.

Historically, visual inspection of the cervix without magnification was the first method of screening of the cervix. Currently, three different types of tests are promoted:

- Conventional Pap smear (or cytology) and liquid-based cytology

- Visual inspection with Acetic Acid (VIA) or with lugol iodine (VILI)

- HPV testing for high risk HPV types (e.g., types 16 and 18).

The randomised trials on cervical cancer screening have all been conducted in India, and have documented the efficacy of visual inspection, cytology screening and HPV-screening [18-21]. Numerous epidemiological studies have consistently documented that in countries where the resources exist to ensure high-quality and good coverage of the population, cytology screening contributes to decreasing the incidence of advanced-stage cancers and mortality associated with cervical cancer [22-24].

A priori, VIA or with VILI are the screening methods that would be the most cost-effective in African environments. VIA and VILI are performed in women who are 30 years up to 50 years. Women younger than 30 years should not undergo screening except for women known to be HIV-infected or living in a high HIV prevalence area. 
A screening test followed in the same visit by treatment of positive results is referred to as a 'screen and treat' or 'see and treat' protocol. This approach is only possible with screening tests that produce immediate results (e.g., visual inspection, rapid-result HPV testing). The treatment methods mostly used are cryotherapy, loop electrosurgical excision procedure or cold-knife conisation.

There are also 'Two-visit protocols' which typically include a first visit with cervical cytology followed by a second visit with colposcopy and treatment based on the colposcopic examination. Two-visit protocols should not be used in populations where patients cannot afford (e.g., for economic or logistic reasons) more than one visit to outpatient clinics.

HPV testing detects strains of the virus that are associated with a high risk of cervical neoplasia. There is no commercially available test for detection of low-risk HPV strains. A specimen for HPV testing can be obtained using a Dacron swab or an endocervical brush, or some liquid-based cytology samples can also be used for HPV testing.

There are two types of available HPV tests:

- Tests that detect the presence or absence of any of 13 to 14 high-risk HPV subtypes that are associated with cervical cancer. These tests do not report which of the individual subtypes are present.

- Tests that perform HPV genotyping and report the presence or absence of HPV 16 or 18, such as Hybrid Capture II HPV testing

\section{Screening for cervical cancer in HIV-infected women and adolescents}

Women who are infected with HIV should undergo cervical cancer screening twice in the first year after diagnosis of HIV infection and then annually, provided the test results are normal.

For women with two consecutive normal cytological examinations, the recommendation is that annual follow-up includes a thorough visual inspection of the anus, vulva, and vagina, as well as the cervix.

There is no consensus as to whether HPV testing should be performed routinely on HIV-infected women.

\section{Discussion}

Several methods exist for cervical cancer prevention. All represent complex undertakings, incurring costs and the need of competent human resources.

Primary prevention based on condom use and circumcision are efficient methods. However, there is little spontaneous inclination to accept these methods and adequate information is necessary for disseminating the conviction that one can fight a deadly disease (and STIs in general) with these methods.

Countries contemplating the introduction of screening technologies should not overlook major issues encountered when planning screening activities. A first issue is the need to ensure therapy of screen-detected lesions. In this regard, visual inspection techniques might be the most cost-effective. Moreover, screening could be provided as a priority to HIV-infected women and to women with an STI.

Nationwide implementation of cytology screening or on HPV-detection is presently out of reach for most African countries. These screening technologies are costly and require the training of smear test readers, the shipment of tests, and more than one encounter with screened women, which may be difficult in many settings.

Nationwide HPV vaccination programmes are facing economic constraints, mainly the cost of vaccines which remain a barrier for countries where the health budget is only a few euros per capita per year. However, costs of HPV vaccines tend to steadily decrease, and thus affordability is rising on the horizon.

A recurrent question is whether priority should be given to screening or to HPV vaccination policies. Where screening is rare (i.e., most of Africa), probably anti-HPV prophylaxis should be considered first, as this policy is likely to drastically reduce the burden of HPV-related genital neoplasia on the long term. One way to contemplate screening versus vaccination issues is to conduct cost-effectiveness studies in 
countries where cervical cancer control is on the agenda. Up to now, most economic studies have focused on HPV vaccination considered alone, or in combination with screening like in South-Africa [25-28]. Future economic studies should compare the marginal cost-effectiveness of HPV vaccination against screening using, say, visual inspection according to a one-step strategy. These studies could also address specific issues like young women with HIV infection, for which screening starting at an early age (say around 20 instead of 30 years of age) could remain a valid strategy. Such studies have the potential to provide an exhaustive overview of needs and constrains for each approach in resource-limited settings, and provide metrics for comparing them and make informed choices.

\section{Conclusion}

Sub-Saharan countries have still a long way to go for controlling the high burden of cervical cancer. Effective prevention methods exist, such as screening, HPV vaccination and safe sex behaviours, but their affordability and implementation remain challenging for most of these countries. The perspective of steadily decreasing costs of HPV vaccines is an invitation for examining cost-effective ways to plan vaccination programmes, and consider whether vaccination should not be given the priority.

\section{Conflicts of interest}

None to disclose.

\section{Funding statement}

This work was part of academic duties. No external funding source supported this work.

\section{References}

1. Ferlay J, Soerjomataram I, and Ervik M, et al (2012) GLOBOCAN 2012 v1.1, Cancer Incidence and Mortality Worldwide: IARC CancerBase No. 11 [http://globocan.iarc.fr] Date accessed: 24/06/15

2. Jemal A, Bray F, and Forman D, et al (2012) Cancer burden in Africa and opportunities for prevention Cancer 118(18) 4372-4384 https://doi.org/10.1002/cncr.27410 PMID: 22252462

3. Arbyn M, Castellsague X, and de Sanjose S, et al (2011) Worldwide burden of cervical cancer in 2008 Ann Oncol 22(12) 2675-2686 https://doi.org/10.1093/annonc/mdr015 PMID: 21471563

4. Walboomers JM, Jacobs MV, and Manos MM, et al (1999) Human papillomavirus is a necessary cause of invasive cervical cancer worldwide J Pathol 189(1) 12-19 https://doi.org/10.1002/(SICI)1096-9896(199909)189:1\&lt;12::AID-PATH431\&gt;3.0.CO;2-F PMID: 10451482

5. Li N, Franceschi S, and Howell-Jones R, et al (2011) Human papillomavirus type distribution in 30,848 invasive cervical cancers worldwide: variation by geographical region, histological type and year of publication. Int J Cancer 128(4) 927-935 https://doi.org/10.1002/ijc.25396

6. Chirenje ZM (2005) HIV and cancer of the cervix Best Pract Res Clin Obstet Gynaecol 19(2) 269-276 https://doi.org/10.1016/j.bpobgyn.2004.10.002 PMID: 15778115

7. Seck AC, Faye MA, and Critchlow CW, et al (994) Cervical intraepithelial neoplasia and human papillomavirus infection among Senegalese women seropositive for HIV-1 or HIV-2 or seronegative for HIV Int J STD AIDS 5(3) 189-193 https://doi. org/10.1177/095646249400500307 PMID: 8061090 
8. Gichangi PB, Bwayo J, and Estambale B, et al (2003) Impact of HIV infection on invasive cervical cancer in Kenyan women AIDS 17(13) 1963-1968 https://doi.org/10.1097/00002030-200309050-00015 PMID: 12960829

9. Arbyn M, Xu L, and Simoens C, et al (2018) Prophylactic vaccination against human papillomaviruses to prevent cervical cancer and its precursors Cochrane Database Syst Rev 5 Cd009069 PMID: 29740819 PMCID: 6494566

10. Palmer T, Wallace L, and Pollock KG, et al (2019) Prevalence of cervical disease at age 20 after immunisation with bivalent HPV vaccine at age 12-13 in Scotland: retrospective population study BMJ 365 I1161 https://doi.org/10.1136/bmj.l1161

11. World Health Organization (2014) Human Papillomavirus vaccines: WHO Position Paper (Geneva, Switzerland: World Health Organization)

12. Green EC, Halperin DT, and Nantulya V, et al (2006) Uganda's HIV prevention success: the role of sexual behavior change and the national response AIDS Behav 10(4) 335-346 discussion 47-50 https://doi.org/10.1007/s10461-006-9073-y PMID: 16688475 PMCID: 1544373

13. Baldwin SB, Wallace DR, and Papenfuss MR, et al (2004) Condom use and other factors affecting penile human papillomavirus detection in men attending a sexually transmitted disease clinic Sex Transm Dis 31(10) 601-607 https://doi.org/10.1097/01. olq.0000140012.02703.10 PMID: 15388997

14. Sankaranarayanan $\mathrm{R}$, Ramadas $\mathrm{K}$, and Thara $\mathrm{S}$, et al (2013) Long term effect of visual screening on oral cancer incidence and mortality in a randomized trial in Kerala, India Oral Oncol 49(4) 314-321 https://doi.org/10.1016/j.oraloncology.2012.11.004

15. Sankaranarayanan R, Esmy PO, and Rajkumar R, et al (2007) Effect of visual screening on cervical cancer incidence and mortality in Tamil Nadu, India: a cluster-randomised trial Lancet 370(9585) 398-406 https://doi.org/10.1016/S0140-6736(07)61195-7 PMID: 17679017

16. Shastri SS, Mittra I, and Mishra GA, et al (2014) Effect of VIA screening by primary health workers: randomized controlled study in Mumbai, India J Natl Cancer Inst 106(3) dju009 https://doi.org/10.1093/jnci/dju009 PMID: 24563518 PMCID: 3982783

17. Sankaranarayanan R, Nene BM, and Shastri SS, et al (2009) HPV screening for cervical cancer in rural India New Engl J Med 360(14) 1385-1394 https://doi.org/10.1056/NEJMoa0808516 PMID: 19339719

18. Laara E, Day NE, and Hakama M (1987) Trends in mortality from cervical cancer in the Nordic countries: association with organised screening programmes Lancet 1(8544) 1247-1249 https://doi.org/10.1016/S0140-6736(87)92695-X PMID: 2884378

19. van der Aa MA, Pukkala E, and Coebergh JW, et al (2008) Mass screening programmes and trends in cervical cancer in Finland and the Netherlands Int J Cancer 122(8) 1854-1858 https://doi.org/10.1002/ijc.23276

20. Sigurdsson K and Sigvaldason H (2006) Longitudinal trends in cervical histological lesions (CIN 2-3+): a 25-year overview Acta Obstet Gynecol Scand 85(3) 359-365 https://doi.org/10.1080/00016340500432481 PMID: 16553186

21. Tan N, Sharma M, and Winer R, et al (2018) Model-estimated effectiveness of single dose 9-valent HPV vaccination for HIVpositive and HIV-negative females in South Africa Vaccine 36(32 Pt A) 4830-4836 https://doi.org/10.1016/j.vaccine.2018.02.023 PMID: 29891348 PMCID: 6508597

22. Li X, Stander MP, and Van Kriekinge G, et al (2015) Cost-effectiveness analysis of human papillomavirus vaccination in South Africa accounting for human immunodeficiency virus prevalence BMC Infect Dis 15566 https://doi.org/10.1186/s12879-015-1295-z PMID: 26652918 PMCID: 4676856

23. Sinanovic E, Moodley J, and Barone MA, et al (2009) The potential cost-effectiveness of adding a human papillomavirus vaccine to the cervical cancer screening programme in South Africa Vaccine 27(44) 6196-6202 https://doi.org/10.1016/j.vaccine.2009.08.004 PMID: 19698807

24. Tracy JK, Schluterman NH, and Greene C, et al (2014) Planning for human papillomavirus (HPV) vaccination in sub-Saharan Africa: a modeling-based approach Vaccine 32(26) 3316-3322 https://doi.org/10.1016/j.vaccine.2014.03.067 PMID: 2473173 